\title{
The clinical significance of IL-6s and IL-27s in Bronchoalveolar lavage fluids from children with mycoplasma pneumoniae pneumonia
}

Jie Zhao

Zibo Central Hospital

Yuyun Li

Zibo Central Hospital

Wen Zhang ( $\sim$ zangyua@yeah.net)

Research article

Keywords: mycoplasma pneumoniae pneumonia; Bronchoalveolar lavage fluid; IL-6; IL-27; communityacquired pneumonia

Posted Date: October 9th, 2019

DOI: https://doi.org/10.21203/rs.2.15861/v1

License: (c) (i) This work is licensed under a Creative Commons Attribution 4.0 International License. Read Full License 


\section{Abstract}

Backgrounds: IL-6 is associated with the severity of mycoplasma pneumoniae pneumonia (MPP). But the relationship of IL-27 and MPP was unknown. Methods: 98 patients with MPP younger than 14 years old were enrolled in this study and divided into mild cases and severe cases by severity of disease, MP single infection group and MP mixed infection group by MP mixed-infection types, and low MP DNA loads group and high MP DNA loads group by MP DNA loads respectively. 15 children with foreign bronchus bodies were also enrolled as control. The levels of IL- 6 and IL-27 in BALFs collected from these children were assayed by ELISA. Results: There were significant differences in the levels of IL-6 in BALFs from patients between 3 patient groups, respectively ( $\mathrm{P} \otimes 0.05$ ). Compared with the levels of IL-6 in BALFs from control, the levels of IL- 6 in BALFs from the 5 groups were significantly higher ( $\mathrm{P} \otimes 0.05)$ except that from low MP DNA loads group. The levels of IL-27 in BALFs from MP mixed infection group were significantly higher than those from MP single infection group and control( $P \otimes 0.05)$. Conclusion: IL-6 was firmly associated with MPP and had potential application in clinical practice while IL-27 was not associated with MP infection.

\section{Background}

Mycoplasma pneumoniae (MP) is a main pathogen in respiratory infections in children. It causes more than $40 \%$ of community-acquired pneumonia (CAP) cases in children and $18 \%$ of these cases needs hospitalization [1]. At present there have been many problems with patients suffering from Mycoplasma pneumoniae pneumonia (MPP) such as the increasing macrolide resistance rate ${ }^{[2]}$, the complex multiple systemic complications ${ }^{[3]}$, the increasing occurrence of refractory MPP ${ }^{[4]}$. Therefore, MPP has attracted the great attention of many researchers and patients. It is widely recognized that immune function disorders have been involved in the pathogenesis of MPP [5]. And IL-6 is an important cytokine which can regulate immune function ${ }^{[6]}$. It was involved in the infection process of $M P$ and played an important role in the pathogenesis of MPP [7]. One study suggested that IL-6 was associated with the severity of MPP [8]. IL-27 could induce the secretion of IL-6 ${ }^{[9]}$ and block the activity of IL- 6 by its subunit of IL-27 p28 ${ }^{[10]}$. Therefore, we inferred that IL-27 might be involved in the pathogenesis of MPP.

Fiberoptic bronchoscopy and bronchoalveolar lavage is safe and effective in the diagnosis and treatment of MPP, which can provide bronchoalveolar lavage fluids (BALFs) for research. BALFs can reflect the pathological and biochemical changes of lung tissues directly. However, there have been few reports about IL- 6 and IL-27 in BALFs from MPP patients. Only a few reports about the relationship between IL- 6 in sera and the severity of MPP ${ }^{[8]}$ can be found.

In this study, the changes of IL-6 and IL-27 in BALFs from MPP patients and control were assayed to explore their clinical significances.

\section{Methods}


In this study, the diagnosis of MPP met the following criteria: 1) fever, coughing, and other respiratory tract infection symptoms; 2) chest radiographic examination with bronchial pneumonia, interstitial pneumonia, segmental or lobar pneumonia, and even pleural effusion; and 3) a single serum anti- MPIgM antibody titers of $\geq 1: 160$ at the acute phase following admission (in those with no history of respiratory infections in the past 3 months) and a positive PCR test for MP. Patients with any of the following criteria would be diagnosed as severe cases: 1 ) tachypnea ( $\geq 40 / \mathrm{min}$ ) or tachycardia ( $\geq 120 / \mathrm{min}) ; 2$ ) hypoxemia (PaO2<60mmHg); 3) multilobar involvement or involvement area $\geq 2 / 3$ on chest radiographs; 4 ) pleural effusion $(>300 \mathrm{ml})$ and atelectasis. Patients having infections within 3 months, or suffering from known coexisting chronic, progressive or oncological illnesses, or receiving corticosteroids or immunosuppressive agents within 3 months, or with immune hypofunction or immune related diseases, or allergic diseases were excluded from the research.

All the patients were divided into severe cases and mild cases by the severity of the diseases. The whole patients could also be divided into MP single infection group and MP mixed infection group according to

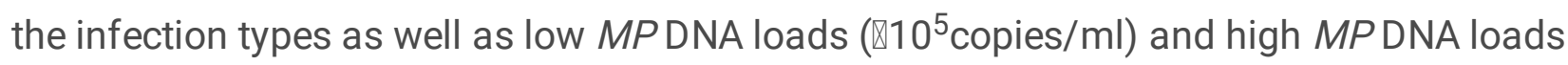
$\left(\geq 10^{5}\right.$ copies $/ \mathrm{ml}$ ) according to the MP DNA loads.

\subsection{Data collection}

Data including age, gender, clinical signs and symptoms, laboratory and radiological findings were collected. All chest radiographs were reviewed by two experienced radiologist and they agreed on the conclusion.

\subsection{Collection of BALFs}

Bronchoscopy was performed within 3 days after hospital admission for patients with MPP and immediately after hospital admission to remove the foreign bronchus bodies for children in control. Flexible fiber optic bronchoscopy and bronchoalveolar lavage were performed following the guidlines described previously ${ }^{[11]}$. BALFs were collected from these children and stored in $-80^{\circ} \mathrm{C}$ freezer.

\subsection{The assay of IL-6 and IL-27}

The levels of IL- 6 and IL-27 in BALFs of the children were assayed with kits by the method of double antibody sandwich ELISA. The kits were offered by Abcan Company. The experiments were strictly conducted in accordance with the manufacturer's instructions.

\subsection{Statistical analysis}

Statistical analyses were performed using SPSS22.0 Statistical package. Continuous variables are reported as the mean \pm standard deviation. ANOVA were used to compare means of multiple groups. LSD 
test were used for the inter-comparison of 2 means in the multiple groups. $P \llbracket 0.05$ was considered to indicate a statistically significant difference.

\section{Results}

\subsection{General information}

From Jan $1^{\text {st }}$ to Dec $31^{\text {st }}$ of the year 2017, a total of 98 hospitalized children with MPP according to the including criteria and excluding criteria were enrolled. All patients including 47 male patients and 51 female ones underwent fiberoptic bronchoscopy and BALFs were collected from them. The age of the patients ranged from 1 to 13 years $(6.17 \pm 2.32$ years). During the study period, 15 children with foreign bronchus bodies consisting of 8 males and 7 females were also enrolled in this research as control. The ages of them were among 1 to 9 years ( $4.97 \pm 3.15$ years). There were no inflammatory changes on the chest radiographs of children in control. No significant differences in ages $(t=1.77, p \otimes 0.05)$ and sex ratio $\left(x^{2}=0.15, p \otimes 0.05\right)$ between the patients and control were observed.

The patients were divided into severe cases and mild cases by severity of disease. Severe cases consisted of 27 male patients and 28 female ones. The age of them was $6.83 \pm 2.61$ years old. Mild cases included 43 patients with 20 boys and 23 girls. The age of them averaged $5.80 \pm 2.82$ years old. The whole patients were grouped into MP single infections (60 cases) and MP mixed infections (38 cases) according to the infection types. MP single infections consisted of 25 male patients and 35 female ones. The age of them was $6.46 \pm 2.73$ years old. MP mixed infections included 38 patients with 22 boys and 16 girls. The age of them averaged $5.97 \pm 2.79$ years old. The patients could also be divided into low MPDNA loads $\left(1.0 \times 10^{3}\right.$ copies $/ \mathrm{ml} \leq 50$ cases $\otimes 10^{5}$ copies $\left./ \mathrm{ml}\right)$ and high MP DNA loads ( 48 cases $\geq 10^{5}$ copies $/ \mathrm{ml}$ ) according to the MPDNA loads. Low MPDNA loads consisted of 23 male patients and 27 female ones. The age of them was $6.92 \pm 2.86$ years old. High MPDNA loads included 48 patients with 24 boys and 24 girls. The age of them averaged $5.86 \pm 2.67$ years old. No significant differences in ages $(t=1.87, p \otimes 0.05$; $\mathrm{t}=0.86, \mathrm{p} \otimes 0.05 ; \mathrm{t}=1.89, \mathrm{p} \otimes 0.05)$ and sex ratio $\left(\mathrm{x}^{2}=0.06, \mathrm{p} \otimes 0.05 ; \mathrm{x}^{2}=2.45, \mathrm{p} \otimes 0.05 ; \mathrm{x}^{2}=0.16, \mathrm{p} \otimes 0.05\right)$ were observed between severe cases and mild cases, MP single infections (60 cases) and MP mixed infections as well as low MPDNA loads and high MPDNA loads.

2.2 The comparison of IL-6s and IL-27s in BALFs among severe cases, mild cases and control, respectively

The levels of IL-6 in BALFs from severe cases were higher than those from control and mild cases. There were significant differences between them $(p<0.05)$. The levels of IL- 6 in BALFs from mild cases were significantly higher than those from control $(p<0.05)$. The levels of IL-27 in BALFs from severe cases and mild cases were slightly decreased than those from control, but there were no significant differences in the levels of IL-27 among them ( $p>0.05)$. (Table 1)

2.3 The comparison of IL-6s and IL-27s in BALFs among MP single infection group, MP mixed infection group and control, respectively 
The levels of IL- 6 in BALFs from MP mixed infection group were higher than those from control and MP single infection group. There were significant differences between them $(p<0.05)$. The levels of IL- 6 in BALFs from MP single infection group were significantly higher than those from control $(p<0.05)$. The levels of IL-27 in BALFs from MP mixed infection group were significantly lower than those from control and $M P$ single infection group $(p<0.05)$. But no significant difference were found in the levels of IL-27 in BALFs between MP single infection group and control ( $p>0.05$ ). (Table 2 )

2.4 The comparison of IL-6s and IL-27s in BALFs among high MP DNA loads group, low MP DNA loads group and control, respectively

The levels of IL- 6 in BALFs from high MP DNA loads group were higher than those from control and low MPDNA loads group. There were significant differences between them $(p<0.05)$. The levels of IL- 6 in BALFs from low MP DNA loads group were not significantly higher than those from control $(p>0.05)$. There were not significant differences in the levels of IL-27 in BALFs from high MP DNA loads group, low MPDNA loads group and control ( $p>0.05)$. (Table 3)

\section{Discussion}

MPP is a common respiratory infection in children and a leading cause of death in China ${ }^{[12]}$. In recent years, patients infected by MP have increased year by year in the worldwide ${ }^{[13]}$. So MPP has drawn much attention from medical practitioners. The main clinical manifestations include fever, cough, shortness of breath and continuous dry and wet rales in the lung. Patients often present from mild symptoms to severe symptoms. It was reported that IL-6 was associated with the severity of MPP ${ }^{[8]}$. But no reports about IL-6 relating to MP DNA loads and MP infection types in patients with MPP have been found. There are also no reports found about the relationship of IL-27 and MPP. So the research aimed to explore the relationship of IL- 6 and MPP further as well as the relationship of IL-27 and MPP.

IL-6 is an important cytokine that has dual functions in the process of inflammation ${ }^{[6]}$. It is mainly secreted by Th2 cells and can promote humoral immune responses to extracellular microbial pathogens and the secretion of protective antibodies ${ }^{[6]}$. IL-6 plays an important role in the pathogenesis of MPP [7]. Our previous research suggested that the IL-6-174 G/C genotype increased the infection of MP in patients [14], which also suggested that IL-6 was closely related to MP infection. In the research, the level of IL-6 was significantly higher in patients with MPP than those in control. There were significant differences in the levels of IL- 6 between severe cases and mild cases. It suggested that IL-6 was closely related to the severity of the disease, which was similar to the previous report ${ }^{[8]}$. The levels of IL-6 in MP mixed infection group was significantly higher than that in MP single infection group, which suggested that IL-6 may be associated with other pathogens ${ }^{[15]}$. IL-6 was closely associated with MP DNA loads for there were significant difference in the levels of IL-6 between high MP DNA loads group and low MPDNA loads group. These results have not been found reported previously. Additionally, IL-6 in RMPP patients was significant higher than that in the General MPP patients ${ }^{[16]}$. IL-6 was also associated with pleural effusion ${ }^{[17]}$ and radiological appearance ${ }^{[8]}$ in MPP patients. Guo $L$ et al reported that levels of IL-6 in serum of 
patients infected by the macrolide-resistant strains were higher than those infected by non macrolideresistant strains ${ }^{[18]}$. Therefore IL-6 was closely related to MPP. In the future, it may be used as an indicator for reflecting the severity and the infection state of the disease.

IL-27 is produced by antigen-presenting cells upon exposure to microbial-derived molecules and inflammatory stimuli ${ }^{[19]}$. IL-27 has emerged as a pro-inflammatory factor that signals via binding to IL27R, which consists of IL-27Ra (WSX-1/TCCR) and glycoprotein 130 subunits, and mediates various inflammation-promoting biological activities involved in the pathogenesis of many inflammation-related diseases ${ }^{[20]}$. Increasing evidence suggested that IL-27 was a strong inducer of chemokines and proinflammatory cytokines including IL-6 by activated neutrophils, monocytes, and macrophages ${ }^{[9]}$. IL-27 is a heterodimeric cytokine constituted of two subunits, EBI3 and IL-27-p28 [21], and IL-27 p28 can blocks the activity of IL-6 ${ }^{[10]}$. Therefore, IL-27 may maintain the level of IL-6 in a balance state.

In this research, there were no significant differences in the level of IL-27 between MPP groups and control, MP severe cases and MP mild cases, high MPDNA loads and low MP DNA loads, which suggested that IL-27 was not related to MP infection. However, the levels of IL-27 in MP mixed infection group was significantly lower than that in MP single infection group, which suggested that IL-27 may be related with other pathogens. Therefore, it was postulated that IL-27 was not involved in the pathogenesis of MPP.

However, the research still has some limitations. The samples size included in the study was not large enough. The pathogens in the MP mixed infection group included many kinds of pathogens such as respiratory syncytial virus, chlamydia pneumonia, influenza $A$ virus. The number of each kind of pathogens coinfected in the MP mixed infection group was too few to get a statistical analysis. Therefore, the association of IL-6, IL-27 and other pathogens was hard to analysis.

\section{Conclusions}

In summary, IL-6 was closely related with the severity, MPDNA loads, and MP mixed infections in the patients with MPP. It was a potential indicator in clinical practice. However, IL-27 was not related to MP infections but may be related to other pathogens. So they both should be studied further.

\section{Abbreviations}

MPP mycoplasma pneumoniae pneumonia

MP mycoplasma pneumoniae

BALFs bronchoalveolar lavage fluids

CAP community-acquired pneumonia 


\section{Declarations}

Competing interests

The authors declare no competing interests.

Acknowledgements

We thank the hospital pediatricians and clinical teams on all the pediatric wards who provide care to the children.

Funding

This research did not receive any specific grant from funding agencies in the public, commercial, or notfor-profit sectors.

Availability of supporting data

The datasets used and/or analyzed during the current study are available from the corresponding author on reasonable request.

Authors' contributions

LYY and ZJ conceptualized the study. LYY and ZJ were responsible for data curation, formal analysis, did the experiment and wrote the original draft. ZW was responsible for resources, supervision, validation, visualization and experiments. All authors read and approved the final manuscript.

Ethics approval and consent to participate

The study followed the principles of the Declaration of Helsinki and was approved by the Ethics Committee of Zibo Central Hospital. Written informed consent was obtained from the guardians of the patients.

Consent for publication

All authors have read and approved the manuscript for publication.

\section{References}

1. Sondergaard MJ, Friis MB, Hansen DS, Jorgensen IM. Clinical manifestations in infants and children with Mycoplasma pneumoniae infection. PLoS One. 2018;13: e0195288.

2. Cheong KN, Chiu SS, Chan BW, To KK, Chan EL, Ho PL. Severe macrolide-resistant Mycoplasma pneumoniae pneumonia associated with macrolide failure. J Microbiol Immunol Infect. 2016; 
49:127-130.

3. 3. Chaudhry R, Ghosh A, Chandolia A. Pathogenesis of Mycoplasma pneumoniae: An update. Indian J Med Microbiol. 2016; 34: 7-16.

4. Inamura N, Miyashita N, Hasegawa S, Kato A, Fukuda Y, Saitoh A, et al. Management of refractory Mycoplasma pneumoniae pneumonia: Utility of measuring serum lactate dehydrogenase level. $J$ of Infect Chemother. 2014; 20: 270-273.

5. 5. Saraya T. Mycoplasma pneumoniae infection: Basics. J Gen Fam Med. 2017; 18: 118-125.

6. Akdis M, Aab A, Altunbulakli C, Azkur K, Costa RA, Crameri R, et al. Interleukins (from IL-1 to IL-38), interferons, transforming growth factor beta, and TNF-alpha: Receptors, functions, and roles in diseases. J Allergy Clin Immuno./ 2016; 138: 984-1010.

7. He J, Liu M, Ye Z, Tan T, Liu X, You X, et al. Insights into the pathogenesis of Mycoplasma pneumoniae. Mol Med Rep. 2016; 14: 4030-4036.

8. Hsieh CC, Tang RB, Tsai CH, Chen W. Serum interleukin-6 and tumor necrosis factor-alpha concentrations in children with mycoplasma pneumonia. J Microbiol Immunol Infect. 2001; 34: 109112.

9. Guzzo C, Che Mat NF, Gee K. Interleukin-27 induces a STAT1/3- and NF-kappaB-dependent proinflammatory cytokine profile in human monocytes. J Biol Chem. 2010; 285: 24404-24411.

10. Stumhofer JS, Tait ED, Quinn WJ $3^{\text {rd }}$, Hosken N, Spudy B, Goenka R, et al. A role for IL-27p28 as an antagonist of gp130-mediated signaling. Nat Immunol. 2010; 11: 1119-1126.

11. Wang K, Gao M, Meng F, Li D, Lu R, Wang Y, et al. Transcriptome analysis of bronckoalveolar lavage fluid from children with severe mycoplasma pneumoniae pneumonia reveals novel gene expression and immunodeficiency. Hum Genomics. 2017;11(1):4-4.

12. Tashiro M, Fushimi K, Kawano K, Takazono T, Saijo T, Yamamoto K, et al. Adjunctive corticosteroid therapy for inpatients with Mycoplasma pneumoniae pneumonia. BMC Pulm Med . 2017;17: 219.

13. Qiu L, Wang L, Tan L, Li M, Wu C, Li L, et al. Molecular characterization of genomic DNA in Mycoplasma pneumoniae strains isolated from serious mycoplasma pneumonia cases in 2016, Yunnan, China. Infect Genet Evol. 2018; 58: 125-134.

14. Zhao J, Zhang W, Shen L, Yang X, Liu Y, Gai Z. Association of the ACE, GSTM1, IL-6, NOS3 and CYP1A1 polymorphisms with susceptibility of mycoplasma pneumoniae pneumonia in Chinese Children. Medicine (Baltimore). 2017; 96: e6642 .

15. Dienz O, Rud JG, Eaton SM, Lanthier PA, Burg E, Drew A, et al. Essential role of IL-6 in protection against H1N1 influenza virus by promoting neutrophil survival in the lung. Mucosal Immunol . 2012; 5: 258-266.

16. Zhang Y, Mei S, Zhou Y, Huang M, Dong G, Chen Z. Cytokines as the good predictors of refractory Mycoplasma pneumoniae pneumonia in school-aged children. Sci Rep. 2016; 6: 37037 .

17. Zhao JL, Wang X, Wang YS. Relationships between Th1/Th2 cytokine profiles and chest radiographic manifestations in childhood Mycoplasma pneumoniae pneumonia. Ther Clin Risk Manag. 2016; 12: 1683-1692. 
18. Guo L, Liu F, Lu MP, Zheng Q, Chen ZM. Increased T cell activation in BALF from children with Mycoplasma pneumoniae pneumonia. Pediatr Pulmonol. 2015; 50: 814-819 .

19. Villarino AV, Larkin J 3rd, Saris CJ, Caton AJ, Lucas S, Wong T, et al. Positive and negative regulation of the IL-27 receptor during lymphoid cell activation. J Immunol. 2005; 174: 7684-7691.

20. Neurath MF. New therapies for sepsis: focus on the interleukin (IL)12 family member IL27. Ann Rheum Dis Suppl. 2007; 3: iii29-iii31.

21. Pflanz S, Timans JC, Cheung J, Rosales R, Kanzler H, Gilbert J, et al. IL-27, a heterodimeric cytokine composed of EBI3 and p28 protein, induces proliferation of naive CD4+ T cells. Immunity. 2002; 16: 779-790.

\section{Tables}

Table1. The comparison of IL-6s and IL-27s in BALFs among three groups of children respectively

\begin{tabular}{llll}
\hline Groups & cases & IL-6s $(\mathrm{pg} / \mathrm{ml})$ & IL-27s $(\mathrm{pg} / \mathrm{ml})$ \\
\hline MPP severe cases & 55 & $98.51 \pm 11.13^{\dagger} \square$ & $145.50 \pm 7.73$ \\
MPP mild cases & 43 & $43.43 \pm 6.27^{\dagger}$ & $142.66 \pm 15.73$ \\
control & 15 & $10.15 \pm 2.08$ & $176.60 \pm 14.08$ \\
F value & & 6.32 & 3.63 \\
P & & 0.01 & 0.06
\end{tabular}

Note: “†” signifies that there was significant difference between MPP cases and control. “】” signifies that there was significant difference between MPP severe cases and MPP mild cases.

Table2. The comparison of IL-6s and IL-27s in BALFs among three groups of children respectively

\begin{tabular}{llll}
\hline Groups & cases & IL-6s $(\mathrm{pg} / \mathrm{ml})$ & IL-27s $(\mathrm{pg} / \mathrm{ml})$ \\
\hline MP single infection & 60 & $61.05 \pm 6.32^{\dagger}$ & $163.02 \pm 11.55$ \\
MP mixed infection & 38 & $97.14 \pm 16.79^{\dagger} \square$ & $117.79 \pm 8.24^{\dagger}$ \\
control & 15 & $10.15 \pm 2.08$ & $176.60 \pm 14.08$ \\
F value & & 7.15 & 9.99 \\
P & & 0.01 & 0.002 \\
\hline
\end{tabular}

Note “十” signifies that there was significant difference between MPP cases and control. "】” signifies that there was significant difference between MPP mixed infection group and MPP single infection group.

Table3. The comparison of IL-6s and IL-27s in BALFs among three groups of children respectively 


\begin{tabular}{llll}
\hline groups & cases & IL-6s $(\mathrm{pg} / \mathrm{ml})$ & IL-27s $(\mathrm{pg} / \mathrm{ml})$ \\
\hline high MP DNA loads & 50 & $101.71 \pm 11.31^{\dagger}$ & $142.36 \pm 25.36$ \\
low MP DNA loads & 48 & $41.26 \pm 5.47$ & $142.66 \pm 15.73$ \\
control & 15 & $10.15 \pm 2.08$ & $146.43 \pm 14.13$ \\
F value & & 7.87 & 0.48 \\
P & & 0.006 & 0.49 \\
\hline
\end{tabular}

Note “†” signifies that there was significant difference between MPP cases and control. “"” signifies that there was significant difference between high MP DNA loads group and low MP DNA loads group. 